\title{
Research and Practice on Experimental Teaching Scheme of Modern Instrumental Analysis
}

\author{
W.B. ZHU, L. WANG, T. JIN \& H.X. LIU (Corresponding author) \\ Tianjin Agricultural University, China
}

\begin{abstract}
Analyzing the main issues in experimental teaching of current Modern Instrumental Analysis (MIA), the principle and major structure of MIA experimental teaching schematic design was put forward and implemented. The practice was showed that student's learning interests and creativity was motivated while manipulative ability was trained combined with better-off learning theory and knowledge in the class via the new experimental teaching schematic design.
\end{abstract}

KEYWORD: Modern instrumental analysis (MIA); experimental teaching scheme; research and practice.

\section{INTRODUCTION}

With the development of scientific technologies, all varieties of modernized analyzing instruments have been emerged to make the Modern Instrumental Analysis (MIA) gradually widespread applying, which becomes a key research approach and indispensable tool in the fields of industry, agriculture, medicine, drug, environment and modern top technologies such as nanometer materials or genomics. The experimental teaching of MIA course is the key link for the students mastering all kinds of modern instruments and analyzing methods. How to make the teaching contents and methods available for the cultivation of students' practical ability and innovative ability is not only the first important issue in consideration but also an emphasis on the breakthrough and innovation of MIA experimental curriculum teaching.

\section{PROPERTY AND CHARACTERISTIC OF MIA COURSE AND MAIN EXPERIMENTAL PROBLEMS}

\subsection{Property and characteristic of MIA course}

MIA is a professional basic curriculum opening towards the undergraduates in our university. Largescale instrument which is involved in the curriculum has a wide range. The form is in combination with theory teaching and experimental teaching. Taking the experimental teaching as principal whilst theory teaching as auxiliary. MIA theory is comparatively abstract. Most of students fell that theory is obscure to understand. Construction of analytical instruments is complicated. The majority never contact and see the precise instruments. Theory of referred varieties analytical methods is different. Instrumental distinction is more and operational conditions are respectively different. Only depending on the theory teaching link in the class would not meet the teaching objective of well learning MIA course. Thus, the experimental teaching link is rather important as computer learning requires the practice.

\subsection{Main problems in MIA experiments}

MIA experiment is divided by 3 types of verification, comprehensiveness and design. Conventional experimental teaching lay stress on the verification experiment, only focus on the knowledge point verification. Students simply comply in the whole experimental course rather than analyze the failure cause even without design by themselves. They are in a passive, blind status. Learning positivity and initiative are limited which is bad for not just arousing students' interests and enthusiasm but also cultivating their ability of resolving actual problems as well thereby they lack of the ability of creativeness application. Definite problems in MIA experiments are mainly performed in 5 aspects. First, students' practical opportunity is few. Due to the limited experimental condition, the amount of each experimental group is too more to be good for the cultivation of excellent students. Second, students mainly make passive practice, lacking of initiative exploration spirit. Students only know what it is rather than why it is in the experimental course; only know what the instrument can do rather than what it can still do; only know the 
method designated in the book for the sample measurement rather than other methods which are also in use. Third, examination indicators for the experimental achievements are single. Experimental results validity is overemphasized to cause that parts of students breach experimental scientificity and objectivity, highlight the theory but despise the experiment, without preview and preparation before the class, hasty dispose the experimental course and plagiarize the experimental reports after the class. Fourth, lacking of necessary ability training course, namely short of the link of transforming the knowledge melt into the ability. MIA experimental abilities involve of basic operational ability for all kinds of instruments, method selecting ability towards different analytic target, treatment ability for the substantial experimental data and comprehensively using the knowledge design the experiments, analyze and resolve practical issues. Fifth, efficiency of large-scale instruments utilization is low with different extents of resource wasting.

\section{GUIDING CONCEPT OF MIA EXPERIMENTAL TEACHING DESIGN}

MIA experimental teaching schematic design, in which is promoted to establish practical teaching schematic design situation as the outbreak of experimental reform, initiating from the demand of the students and producing practice, taking student as the subject whilst ability as objective, mission or project as carrier. Characterized by Theory-Practice integration, action-oriented teaching, it is to highlight the practicability, technicality and comprehensiveness of MIA curriculum, making the students not only own the ability to use the modern analytical instruments carry out the analysis and test towards agricultural samples but also possess necessary quality, knowledge and skills and problem-solving ability to engage in the modern instruments analysis, skillful master the technologies of modern instruments analysis and test together with the instruments utilization and maintenance, gradually cultivate the students rigorous work style and professional ethics, building overall quality management consciousness in order to lay a solid foundation for the subsequent professional curriculum learning.

\section{MIA EXPERIMENTAL TEACHING SCHEMATIC DESIGN FRAMEWORK AND ORGANIZATIONAL FORM}

\subsection{MIA experimental teaching schematic design framework}

The mode with different level, grading, step by step is adopted in MIA experimental teaching, from easy to difficult, from briefness to comprehensiveness, combined with inside and outside the class. To encourage the students to independently set up the project, to fully motivate students learning activity and initiative, cultivate scientific experimental methods and rigorous working attitude by opening experimental teaching is also used in MIA. The experimental contents are focused on the integration with tradition and modernity, combined with the academic foreland and teaching and new achievements of scientific research. Experimental teaching schematic design framework is divided into 3 abilities, 3 levels and 4 modules.

MIA experimental design is aimed at overall cultivation for 3 abilities of students, namely operational ability, application and development ability, research and innovation ability. Therefore, the teacher is required to change previous step-bystep teaching mode. People oriented, fully motivate the students' enthusiasm and initiative for knowledge acquiring, making students alter from "let me do" to "I do". In addition to this, let students freely ponder, analyze and resolve the problem themselves in the experimental design and operational guide; let students walk away from the cycle of conventional idea rather than blindly follow others viewpoints, dare to seek for the innovation and difference for put forward the new idea; lead the students issue and resolve the questions via repeated observation and analysis on experimental phenomena, cultivate keen instinct faculty, innovative thinking method and the spirit of probing the unknown with dare to be innovative.

Experimental teaching is the key link of cultivate the students the ability in combination with theory and practice together with analyze and resolve the actual problem. It can make the students not only master the basic skill of experimental operation but also cultivate them the manipulative ability, innovative ability, design idea, pioneering consciousness and scientific quality. To cultivate students operational ability in the basic skill level, to train them the application and development ability in the comprehensive design level, to culture them the research innovation ability in the technical innovative level. Basic technical level experiment is aimed at promote the students understand the fundamental theory, master correct operational methods, cultivate the students meticulous learning attitude, focus on the combination with professional theory lessons, culture the students fundamental operational ability for laying solid foundation. The purpose of comprehensive design level experiment is to urge the students to put their knowledge into practice, cultivate the students' application and development ability. It is focused on cultivate the students' ability of comprehensively use the professional knowledge analyze and resolve actual problems. 
In the course of comprehensive experiment, it is necessary to help the students to gradually deepen the understanding towards the theory and arouse the idea. For instance, in the experiment of the project about measuring the aliphatic acid in the grease, taking a practical application case (scientific and tech enterprise inspect fatty acid) as the model, we let the students design optimized instrumental analysis condition themselves to appraise the advantages and disadvantages of analysis conditions in terms of the requirement of inspection party. Analyze and resolve the issues by themselves while teacher only offers required guidance. The fundamental purpose of scientific tech innovative level experiment is to cultivate students' research innovation ability, mainly with respect to parts of good students particular in opening to the senior students for the improvement of students overall quality. On one hand, teacher could design to use the experimental scheme with new tech and method, opening students' active idea and brave innovation. On the other hand, we can let the students participate to the teachers' scientific research. Teacher refers experimental project while students issue the experimental scheme, install and modulate experimental facility and test with data treatment by themselves. For example, using the plant extract restrain the nitrosamine yielding in the meat and determine the content via the gas chromatographmass spectrometer.

Finishing of teaching objective plays an important role to enhance the students' quality. Experimental teaching objective is to adapt to the innovative educational requirement towards the talent cultivation, to highlight the cultivation for the students' thinking ability, scientific research ability and innovative ability. So, we need to establish the experimental teaching contents facing to the market requirement, shooting the arrow at the target. For adapt to the requirement from the society towards talents cultivation, teacher should make the experimental contents own the representative and advancement that mix together with the scientific research projects as possible as they could when design the experimental contents.

Meanwhile current new technologies and trends should be combined. Therefore, module structure is used to detect the substance during the whole curriculum experiment in line with different apparatus analysis methods. 4 teaching sub-modules are established including Atomic Absorption Spectroscopy (AAS), Gas Chromatographic (GC), High Performance Liquid Chromatography (HPLC) and Infrared Absorption Spectroscopy (IR). Between each module is either relatively independent or intercoordinated. Comprehensiveness and expansion experimental teaching in each module ensures the relevance and interaction among the modules. Meanwhile, based on mutual characteristics of agricultural samples analysis in different modules and developing frontier, many optional experiment subjects are set in the platform of innovative practice.

\subsection{Organizational form in MIA curriculum experiment design}

Above all, acknowledge relevant laboratory in each sub-module of experimental teaching schematic design in MIA curriculum is taken as the beginning. To import 5S management, to cultivate the students build the criteria, neat lab working environment and scientific working quality. Then, let the students finish the operation in the lab based on learning basic operation of correspondent analyzing instruments. Meanwhile permeate the teaching on basic theory and knowledge and also the operational skill training. Once students master certain skills, technical theory knowledge should be taught. Students should finish the schematic determination and testing for the comprehensive projects of each sub-module in finally. Next, use the teaching mode of 1 team with 2 people, fully utilize and assembly practical teaching resource, to carry out the big cycle via dividing the students into groups together with different sub-module teaching by different teachers. And then cross complete the teaching on other submodules. Ensure that everyone has an instrument with full practical training time.

\section{APPLICATION ACHIEVEMENTS}

\subsection{Set up new theory of MIA experimental teaching}

Teachers accept an impact and baptism ideally more or less conscientious and unselfconscious during the participation via the implementation of MIA experimental design. Course teachers vividly realize only overall strengthen MIA experimental teaching, put the cultivation requirements for the vocational ability and innovative consciousness running through the educational teach link and everyone's thought and behavior, application talents would be cultivated that available for the society. Besides, all the course teachers are promoted to better pour into the exploration and practice in MIA experimental teaching themselves, hard enhance practical teaching ability and level, actively probe into the teaching modes and methods which are conformed to the talents cultivation rules in our university, via the operation of each project module.

\subsection{Increase the confidence from students towards MIA curriculum learning}

One of the roots for the students who are weary of learning with low confidence in our teaching is that current teaching contents and modes are broken away from the students. Via the reform of MIA 
experimental teaching contents and modes, students are willing to learn. Students' feedback advice is generally reflected after the class that this teaching mode owned rich independent exploration atmosphere. Students' activity for the experiment is high to not just availably profound the understanding towards the theory knowledge but apparently strengthen the comprehensive applying ability and information retrieval. Students are deeply aware of "on paper will sleep shallow, know this matter to practice". Some other students write articles that comprehensive experimental design in this module is beneficial for us so that we not only learn more knowledge beyond the book but also see our shortage as well as arouse our rich interests towards MIA experiment and feasibly cultivate the team spirit after measuring the fatty acid in the grease.

Consequently, MIA experimental teaching schematic design and practice is the preliminary attempt in the MIA experimental teaching reform. Though achieving certain effect, many practical exploration and educational idea thought are in need for how to further improve and disseminate. We will strive for the better achievements in the future. We also believe that students interests for probing into the science and innovative spirit will be cultivated and enhanced endeavored with the teachers and students in common.

\section{REFERENCES}

[1] Yong, H. 2013. How to Stimulate Students' Study Interest in the Course of Instrument Analysis. Guangzhou Chemical Industry, 2: 063.

[2] Lei M, XianFu Z. \& AiMin N. 2012. Application of interest teaching method in teaching of instrument analysis of non-chemistry major. Acta Agriculturae Jiangxi, 24(2): 201-203.

[3] Fanglou L, Chao Y.\&Gen-sheng DOU. 2011. Teaching Innovation of the Course of Instrument Analysis on Tobacco. Journal of Anyang Institute of Technology, 2: 035 .

[4] Weiju Z, Cun L. \& Zhenyu W. 2013.Exploration and Practice on Designing Experiments in Instrument Analysis Chemistry. Guangdong Chemical Industry, 10: 091.

[5] Junjie W, Jinqiang H. \& Yan W. 2013. Discussion on Curriculum Reform of Education, Science, Research Integration in Environmental Instrument Analysis. Guangdong Chemical Industry, 4: 079. 